\title{
Identificação e quantificação de metal pesado em formulações minerais comercializadas no Estado de São Paulo
}

\author{
Identification and quantification of heavy metal in mineral salt \\ commercialized in São Paulo State, Brazil
}

\section{Identificación y cuantificación de metal pesado en formulaciones minerales comercializadas en el Estado de São Paulo, Brasil}

\section{Wilmar Sachetin Marçal'; Laurenil Gaste'; Paulo Eduardo Pardo²; Otávio Campos Neto3; Marcos Roberto Lopes do Nascimento ${ }^{4}$}

Departamento de Clínicas Veterinárias da Universidade Estadual de Londrina, Londrina, PR.

\section{Resumo}

Objetivo: Quantificar o elemento chumbo em diferentes formulações minerais comercializadas no Estado de São Paulo. Material e Método: As amostras de sal mineral foram coletadas diretamente do estoque de estabelecimentos comerciais, durante os anos de 2001 e 2002. Com aproximadamente 200 gramas, foram analisadas pela técnica de espectrofotometria de absorção atômica, no Laboratório de Poços de Caldas, MG, da Comissão Nacional de Energia Nuclear (CNEN). Resultados: Em 24 amostras analisadas, 16 tiveram valores superiores aos 10 ppm recomendados como máximo valor aceitável. Os números oscilaram entre 3,0 e $156 \mathrm{mg} \mathrm{kg}^{-1}$. O maior resultado foi encontrado numa amostra de sal mineral colhida no município de Santo Anastácio, SP. Conclusões: Os resultados demonstram a necessidade do monitoramento junto aos fabricantes, pois algumas misturas minerais podem ser perigosas, possibilitando efeitos cumulativos tóxicos de chumbo inorgânico aos bovinos.

Palavras-chave: Nutrição animal. Suplementos minerais. Cloreto de sódio. Metal pesado. Contaminação por chumbo. Espectrofotometria atômica. Bovinos.

'Professor Associado do Departamento de Clínicas Veterinárias da Universidade Estadual de Londrina, Londrina, PR. CRMV/PR 2269 e CRMV/PR 0743 ${ }^{2}$ Professor da UNOESTE, Presidente Prudente, SP. CRMV/SP 5970

${ }^{3}$ Professor Titular da Faculdade de Medicina Veterinária e Zootecnia, Garça, SP. CRMV/SP 0250

${ }^{4}$ Químico da Comissão Nacional de Energia Nuclear - CNEN 


\section{Introdução}

A comercialização de sal mineralizado para o consumo animal no Brasil, particularmente em alguns estados onde a pecuária é bem desenvolvida e tecnificada, como é o caso do Estado de São Paulo, representa uma significativa parcela de dividendos nos agronegócios. Por esse motivo, as indústrias produtoras e/ou misturadoras, visando baratear custos para ganhar mercado e garantir suas vendas, utilizam fontes de matérias-primas escolhidas pelo preço mais acessível, inclusive aquelas advindas de importação.

A dicotomia preço e qualidade é uma constante preocupação entre nutricionistas, clínicos veterinários e técnicos voltados à saúde e produção animal. Entretanto, acredita-se que algumas novas formulações minerais possam estar contaminadas por elementos tóxicos, sobretudo metais pesados e substâncias radioativas. Essa suspeita, aliada ao fato de que o governo brasileiro, por meio do Ministério da Agricultura, Pecuária e Abastecimento (Portaria SRD no 20 de 06/07/1997), liberou o uso de fontes alternativas de fósforo a partir de fosfatos de rochas, reitera a necessidade de monitorarem-se as formulações minerais destinadas ao consumo animal no país.

Segundo o próprio Ministério da Agricultura, Pecuária e Abastecimento, existem no mercado nacional aproximadamente 5.500 diferentes misturas minerais, as quais são amplamente comercializadas. No Estado de São Paulo, este número chega a 1.376 diferentes marcas e formulações ${ }^{1}$.
Neste contexto, o objetivo da presente pesquisa foi investigar a presença de metais pesados em diferentes suplementos minerais comercializados no Estado de São Paulo, buscando, com as análises laboratoriais, quantificar contaminantes que possam estar agregados aos elementos minerais, nas formulações preparadas para alimentação animal.

O primeiro elemento escolhido para esse estudo investigativo foi o chumbo, considerado por muitos estudiosos como o elemento químico inorgânico de maior risco à saúde dos animais de criação, particularmente na espécie bovina ${ }^{2-7}$, sendo possível sua veiculação aos animais pela ingestão de formulações minerais comprometi$\operatorname{das}^{1,7-12}$.

Do ponto de vista econômico, diferentes autores destacam que os metais pesados, em particular o chumbo, quando presentes em suplementos alimentares para animais podem causar alterações orgânicas importantes, modificando a performance dos

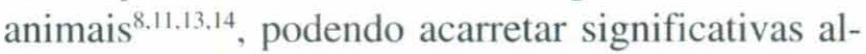
terações no sistema reprodutivo dos bovinos, inclusive abortamento ${ }^{15-17}$.

Existe grande preocupação de pesquisadores, em vários locais do mundo, com a possibilidade de uma formulação mineral contaminada com metais pesados, resultar em uma cadeia trófica comprometida, atingindo os bovinos, e por conseguinte o homem ${ }^{9-14}$. Representa, em larga escala riscos à saúde pública pelo consumo de produtos e subprodutos de origem animal contamina$\operatorname{dos} 1,9,11,18,19$. 


\section{Material e Método}

\section{Coleta e análise das amostras de sal mineral}

As amostras de sal mineral foram coletadas diretamente do estoque disponível em estabelecimentos comerciais, revendedores das formulações já prontas para consumo animal do Estado de São Paulo, durante os anos de 2001 e 2002. As amostras foram acondicionadas em recipientes de plástico transparente, previamente identificados, com aproximadamente 200 gramas de cada diferente marca de produto. As análises foram efetuadas no Laboratório de Poços de Caldas, MG, da Comissão Nacional de Energia Nuclear (CNEN).

Na metodologia analítica empregada para a determinação de chumbo no sal mineral, as amostras foram previamente secas a $110^{\circ} \mathrm{C}$ por aproximadamente duas horas. A solubilização foi feita com os ácidos nítrico, perclórico e fluorídrico. Procedeu-se à determinação do metal por espectrometria de absorção atômica, empregandose um equipamento Varian, modelo 220 FS. Devido à precisão do aparelho nas mensurações, para teores abaixo de $10 \mathrm{mg} \mathrm{kg}^{-1}$, o chumbo era separado da amostra por extração com pirrolidina ditiocarbamato de amônia (APDC) p.a. em $\mathrm{pH} 2,3 \pm 0,1$.

A metodologia de análise empregada baseiase no manual da American Society for Testing and Materials $^{20}$ (1980) e na descrição de Eaton et al. ${ }^{21}$ (1995). O limite mínimo de determinação do método é de $1,5 \mathrm{mg} \mathrm{kg}^{-1}$.

\section{Resultados}

Os resultados obtidos na presente pesquisa, são apresentados na Tabela 1.
Tabela 1 - Resultados da análise laboratorial para quantificação do elemento chumbo inorgânico em diferentes marcas de sal mineral comercializadas no Estado de São Paulo, em 2001 e 2002

\begin{tabular}{|c|c|c|}
\hline $\begin{array}{c}\mathrm{N}^{\mathrm{e}} \text { da } \\
\text { amostra }\end{array}$ & $\begin{array}{c}\text { Município } \\
\text { paulista }\end{array}$ & $\begin{array}{l}\text { Resultado } \\
\text { em mg kg }\end{array}$ \\
\hline 01 & Araçatuba & $4,3 \pm 0,4$ \\
\hline 02 & Avaré & $3,8 \pm 0,4$ \\
\hline 03 & Avaré & $15,4 \pm 1,3$ \\
\hline 04 & Avaré & $35,7 \pm 2,8$ \\
\hline 05 & Avaré & $3,4 \pm 0,3$ \\
\hline 06 & Batatais & $20,2 \pm 1,0$ \\
\hline 07 & Bauru & $9,9 \pm 0,8$ \\
\hline 08 & Birigüi & $13,7 \pm 0,7$ \\
\hline 09 & Birigüi & $18,2 \pm 0,9$ \\
\hline 10 & Campinas & $146,0 \pm 7,0$ \\
\hline 11 & Castilho & $5,6 \pm 0,4$ \\
\hline 12 & Fernandópolis & $16,0 \pm 0,8$ \\
\hline 13 & General Salgado & $26,2 \pm 1,3$ \\
\hline 14 & Martinópolis & $28,2 \pm 1,4$ \\
\hline 15 & Martinópolis & $19,1 \pm 0,9$ \\
\hline 16 & Mogi Mirim & $50,8 \pm 2,5$ \\
\hline 17 & Piracicaba & $28 ; 7 \pm 1,4$ \\
\hline 18 & Presidente Bernardes & $5,0 \pm 0,4$ \\
\hline 19 & Presidente Bernardes & $3,1 \pm 0,2$ \\
\hline 20 & Presidente Prudente & $27,6 \pm 1,3$ \\
\hline 21 & Ribeirão Preto & $3,0 \pm 0,3$ \\
\hline 22 & Santo Anastácio & $156,0 \pm 7,6$ \\
\hline 23 & São Paulo & $34,1 \pm 1,7$ \\
\hline 24 & Taciba & $43,8 \pm 2,2$ \\
\hline
\end{tabular}

\section{Discussão}

A preocupação com formulações minerais contaminadas por elementos metálicos e/ou substâncias radioativas tem sido um desafio constante para técnicos e criadores, originando debates entre pesquisadores em vários países do mundo. 
Também no Brasil, a questão do controle sanitário na alimentação animal tem evoluído muito e se vê, cada vez mais, fortalecida pela participação de adeptos e estudiosos com objetivos práticos. Os recentes episódios da síndrome da vaca louca e da febre aftosa na Europa colocaram o Brasil numa condição especial como exportador de carne. Por isso, monitorar as fontes de matérias-primas que passarão a compor a nutrição mineral dos bovinos é de fundamental importância nesse momento para a pecuária brasileira.

Nesse aspecto, a proposta deste estudo foi investigar a presença do elemento chumbo nos suplementos minerais misturados e produzidos no país, visando contribuir com ações de rastreabilidade e produção natural de bovinos no Brasil, coadunando com a importante e atual ferramenta de marketing às exportações brasileiras, estas, caracterizadas pela disponibilidade do "boi verde". Até então, não havia trabalho desta natureza, considerando as formulações mais comercializadas no Estado de São Paulo. Assim, ressaltou-se a necessidade dessa investigação, já que o Ministério da Agricultura, Pecuária e Abastecimento, ao que se sabe, oficiosamente não detém instrumento prático de fluxo contínuo que atenda a esse objetivo ${ }^{15}$.

Os resultados encontrados na análise laboratorial das amostras de formulações minerais, destacados na Tabela 1 , indicam que em 16 amostras os valores extrapolam o limite máximo aceitável de $10 \mathrm{mg} \mathrm{kg}^{-1}$ preconizado por Maletto ${ }^{11}$ (1986). As publicações originadas do National Research Council $^{5}$ (1980) referendam $30 \mathrm{mg} \mathrm{kg}^{-1}$ como valor máximo aceitável, inclusive mencionando que este limite deve ser considerado na dieta total dos animais. Entretanto, é preciso destacar que, à época de referência deste valor, os equipamentos analíticos não tinham a precisão de hoje, decorridos 24 anos. Além disso, tratando-se de um contaminante, não se deve correr risco e atribuir como parâmetro a dieta total, o que significa valorizar conceitos paliativos que deveriam ter uma importância menor quando se trata de metais pesa- dos atingindo animais e consumidores. Por estas razões, entende-se ser plausível referendar e ter como base de comparação os $10 \mathrm{mg} \mathrm{kg}^{-1}$ como limite máximo, conforme preceitua Maletto ${ }^{11}$ (1986).

Nestas investigações sobre o chumbo foram utilizadas as formulações minerais já misturadas, porque não foi possível separar as matérias-primas para investigar cada um de seus componentes. Suspeita-se que o chumbo na mistura advenha das fontes de fósforo, que se dá porque aquelas representam o maior custo na composição de um sal mine$\mathrm{ral}^{22,23}$, induzindo os fabricantes a buscarem o fosfato para a mistura em fontes alternativas mais baratas. A possibilidade de o chumbo estar agregado às fontes de fosfato, como por exemplo nos fosfatos naturais de rocha ${ }^{7,22,24,25}$ ou no ácido fosfórico importado ${ }^{26}$, certamente pode direcionar pesquisas de investigação, muito embora deva-se lembrar que os metais pesados podem ser oriundos de matérias-primas de microelementos ${ }^{9}$.

É preciso considerar que há aspectos subclínicos ou silenciosos nessa questão de metais pesados e que devem ser lembrados como alerta: os bovinos que consomem sal mineral com níveis considerados tóxicos podem ter alterações no sistema reprodutivo, como anestro, aumento no intervalo entre-partos e alterações de performances, como referem Stuart e Oehme ${ }^{17}$ (1982) e McDowell $^{16}$ (1985).

Se as indústrias misturadoras de sal mineral não aprimorarem a pureza de matérias-primas na composição de suas formulações, o crescimento da comercialização desses sais acarretará a presença de mais contaminantes na alimentação animal, podendo, infelizmente, atingir o homem, originando pontos negativos ao comércio, sobretudo às exportações.

\section{Conclusões}

A análise dos resultados da presente pesquisa permite as seguintes conclusões: 
- Somente 8 amostras analisadas apresentaram resultados inferiores aos $10 \mathrm{mg} \mathrm{kg}^{-1}$, representando $33 \%$ das formulações investigadas;

- 16 amostras das 24 formulações minerais analisadas, representando $67 \%$ dos suplementos minerais avaliados apresentaram resultados superiores aos $10 \mathrm{mg} \mathrm{kg}^{-1}$, que é o limite máximo aceitável;
- O maior valor encontrado, ou seja, $156 \mathrm{mg} \mathrm{kg}^{-1}$, refere-se a uma formulação mineral comercializada na cidade de Santo Anastácio, SP, região de expressiva pecuária de corte no estado;

- Há sólidos indícios técnico-científicos de que está ocorrendo a ingestão de sal mineral com a presença de chumbo pelos bovinos do Estado de São Paulo.

\section{Abstract}

Objective: To quantify the element lead in different mineral formulations traded in the State of São Paulo. Material and Method: Samples of mineral salt were collected directly from the stock of shops throughout the years of 2001 and 2003. The samples, with approximately 200 grams, were analyzed using the anatomic absorption spectrophotometry technique, in the Laboratory located in the city of Poços de Caldas, MG, belonging to the Comissão Nacional de Energia Nuclear (CNEN) - (National Committee of Nuclear Energy). Results: In 24 samples analyzed, 16 presented values higher than the 10-ppm recommended as the maximum acceptable value. Figures ranged from 3.0 to $156 \mathrm{mg}$ $\mathrm{kg-}^{-}$. Highest result was found in a sample of salt collected in the city of Santo Anastácio, SP. Conclusions: Results have shown the need of monitoring procedures before the manufacturers, as some mineral mixtures may be dangerous, with possibility of inorganic lead toxic cumulative effects in cattle.

Keywords: Animal Nutrition. Mineral supplementation. Salt. Heavy Metal. Lead Poisoning. Atomic Absorption Spectrophotometry. Cattle.

\section{Resumen}

Objetivo: Cuantificar el plomo en diferentes formulaciones minerales comercializadas en el Estado de São Paulo, Brasil. Material y Método: Las muestras de sal mineral fueran obtenidas directamente del stock de los establecimientos comerciales, durante los años de 2001 y 2002. Las muestras, de aproximadamente 200 gramos, se analizaron por medio de la técnica de espectrofotometría de absorción atómica, en el Laboratorio de Poços de Caldas, MG, de la Comisión Nacional de Energía Nuclear (CNEN). Resultados: De las 24 muestras analizadas, 16 presentaron valores superiores a $10 \mathrm{ppm}$ considerado como máximo valor recomendado. Los números variaron de 3,0 a $156 \mathrm{mg} \mathrm{kg}^{-1}$. El resultado más alto fue observado en una muestra de sal mineral obtenida en la ciudad de Santo Anastácio, SP. Conclusiones: Esos resultados demuestran la necesidad de un control cuidadoso junto a los fabricantes, una vez que algunas mezclas minerales pueden ser peligrosas, permitiendo efectos tóxicos cumulativos del plomo inorgánico en el ganado.

Palabras-clave: Nutrición animal. Suplementos minerales. Cloruro de sodio. Metales pesados. Contaminación por plomo. Espectrofotometría atómica. Bovinos. 


\section{Referências}

1. MARÇAL, W.S. et al. Valores sangüíneos de chumbo em bovinos Nelore suplementados com sal mineral naturalmente contaminado por chumbo. Ciência Rural, Santa Maria, v. 28, p. 53-57, 1998.

2. KANEKO, J. Clinical biochemistry of domestic animals. 4. ed. New York: Academic Press, 1989. p. 239-891.

3. MARÇAL, W. S. et al. Lead Concentration in mineral salt mixtures used in beef cattle food supplementation in Brazil. Veterinarski Arhiv, Croatia, v. 69, p. 349-355, 1999.

4. MARÇAL, W. S.; TRUNKL, I. Poluição industrial na zona rural: implicações na saúde pública. In: CONGRESSO BRASILEIRO DE MEDICINA VETERINÁRIA, 23., Olinda, 1994. Anais... Olinda: Sociedade Pernambucana de Medicina Veterinária, 1994. p. 656.

5. NATIONAL RESEARCH COUNCIL. Subcommitte on Mineral Toxicity in Animals. Mineral tolerance of domestic animals. Washington, DC: National Academic of Sciences, 1980. p. 256-276.

6. VILlEGAS-NAVARRO, A. et al. Determination of lead in paired samples of blood and synovial fluid of bovines. Experimenthal and Toxicologic Pathology, v. 45, p. 47-49, 1993.

7. AMMERMAN, C. B. et al. Contaminating elements in mineral supplements and their potential toxicity: a review. Journal of Animal Science, v. 44, p.485-508, 1977.

8. ASSOCIATION OF AMERICAN FEED CONTROL OFFICIALS INCORPORATED. Official guidelines for contaminant levels permitted in mineral feed ingredients. Indiana, 2001. p. 292-293.
9. CAMPOS NETO, O.; MARÇAL W. S. Os fosfatos na nutrição mineral de ruminantes. Revista dos Criadores, São Paulo, v. 66, n. 793, p. 8-10, 1996.

10. JUNQUEIRA, O. M. Metais pesados contaminam carne. Avicultura \& Suinocultura Industrial, São Paulo, n. 38, p. 27-29, 1993.

11. MALETTO, S. Correlação da nutrição mineral e a sanidade. In: SEMINÁRIO SOBRE NUTRIÇÃO MINERAL, 1986, São Paulo. Anais... São Paulo, 1986, 38 p.

12. MAYNARD, L. et al. Elementos inorgânicos e seu metabolismo. In: Nutrição animal. 3. ed. Rio de Janeiro: Freitas Bastos, cap. 3

13. LOBÃO. Mineralização de bovinos de corte. In: SIMPÓSIO SOBRE PECUÁRIA DE CORTE, 1977, Presidente Prudente. Anais... Jaboticabal: UNESP, 1977. p. 120-135.

14. SILVA, S. Plano de ação fiscal sobre fosfato de rocha e outros. Brasília: Ministério da Agricultura, do Abastecimento e da Reforma Agrária, 1993. 21 p.

15. MARÇAL, W. S. et al. Concentration of lead in mineral salt mixtures used as supplements in cattle food. Experimenthal and Toxicologic Pathology, Jena, v. 53, p. 7-9, 2001.

16. McDOWELL, L. R. Nutrition of grazing ruminants in warm climates. Orlando: Academic Press, 1985. p. 182-186.

17. STUART, L. D., OEHME, F. V. Environmental factors bovine and porcine abortion. Veterinary and Human Toxicology, v. 24, p. 435-41, 1982. 
18. ALLEN, J. D. Minerals in animal feed. Industrial Minerals, n. 292, p. 35-39, 1992.

19. ANDRIGUETO, J. M. et al. Os princípios nutritivos e suas finalidades. In: Nutrição animal. 4. ed. São Paulo: Nobel, 1990. p. 189-255.

20. AMERICAN SOCIETY FOR TESTING AND MATERIALS. Water. In: Annual book of ASTM standards. Philadelphia: ASTM, 1980. p. $450-464$.

21. EATON, A. D. et al. Standard methods for the examination of water and wastewater. Washington, DC: APHA, 1995. p. 3-16.

22. ROSA, I. V. Fosfato natural como suplemento de fósforo para bovinos. In: VALLE, E. R. et al. Coletânea de seminários técnicos $\mathbf{1 9 8 6 / 8 8}$. Campo
Grande: EMBRAPA, 1989. p. 59.

23. SOUSA, J. C. Aspectos da suplementação mineral de bovinos de corte. Circular Técnica. Centro Nacional de Pesquisa em Gado de Corte, n. 5, p. 1 50,1981 .

24. CAMPOS NETO, O. Pesquisa esclarece dúvidas sobre déficit na nutrição animal. O Corte, São Paulo, v. 24, p.14, 1992.

25. VIANA, J. A. C. Fontes de sais minerais para bovinos e o desafio de suplementos de fósforo no Brasil. In: SIMPÓSIO SOBRE NUTRIÇÃO DE BOVINOS, 3., 1985, Piracicaba. Anais... Piracicaba: FEALQ, 1985.

26. BRITO, J. Fosfato bicálcico feed grade. Cajati: Serrana, 1993. 17 p. Apostila. 